\title{
Desempenho reprodutivo entre receptoras bovinas não contaminadas e naturalmente infectadas por Neospora caninum, após a transferência de embriões ${ }^{1}$
}

\author{
Luís G.R. Sturaro ${ }^{2,3 *}$, Carlos A. Zanenga ${ }^{3}$, Merlison F. Pedroso ${ }^{3}$, Eduardo G. Palazzi², \\ Roberta K.R. Queiroz ${ }^{2}$ e Magali D’Angelo²
}

\begin{abstract}
Sturaro L.G.R., Zanenga C.A., Pedroso M.F., Palazzi E.G., Queiroz R.K.R. \& D'Angelo M. 2013. [Reproductive performance in bovine receipts non infected and naturally infected with Neospora caninum after embryo transfer.] Desempenho reprodutivo entre receptoras bovinas não contaminadas e naturalmente infectadas por Neospora caninum, após a transferência de embriões. Pesquisa Veterinária Brasileira 33(8):970-974. Laboratório de Biologia Celular, Instituto Biológico, Av. Conselheiro Rodrigues Alves 1252, Vila Mariana, São Paulo, SP 04014-002, Brazil. E-mail: luisgustavo_vet@yahoo.com.br

The use of knowledge in biotechnologies for improving the production of goods and services has advanced significantly. The control of infectious diseases continues to represent the most obstacle to the health of animals. The embryonic loss may be responsible for the increase in economic losses for producers isolated from cattle. Infection with Neospora caninum has emerged as an important reproductive disease and, in several countries, has been diagnosed as the main cause of abortion. The aim of this study was to evaluate the reproductive performance in not contaminated and naturally infected with Neospora caninum bovine receipt after embryos transfer (ET). The choice of animals $(n=395)$ for the experiment was performed by gynecological evaluation, via transrectal palpation. Vaccinations recommended for health protocol followed the ET. Nutritional management was based on the National Research Council (NRC, 1999). The ET of thawed embryos was made after the synchronization of the estrous cycle of the receipts. To evaluate the reproductive performance of transrectal ultrasonography was used. The identification of the positive receipts for Neospora caninum was performed by ELISA and reagents for samples, confirmation by indirect immunofluorescence (IIF). Data were analyzed using Past ${ }^{\circledR}$ and were considered statistically significant $(\mathrm{P}<0.01)$. The results show a rate of pregnant $(\mathrm{n}=191)$ of $48.35 \%$, followed by an abortion rate at 60 days of gestation $(n=14)$ and $3.5 \%$ of abortions in the sixth month of gestation $(n=12)$ of $3.04 \%$. These results become relevant when taking into consideration that infected receipts by the protozoan not showed a high rate of pregnancy loss. The statistical insignificance of the data obtained in this study provides the security suggesting that there is no need to deploy the diagnosis or prior control to neosporosis in receipts able to ET.
\end{abstract}

INDEX TERMS: Neosporosis, Neospora caninum, abortion, conception, embryo receipts, cattle.

\footnotetext{
${ }^{1}$ Recebido em 20 de abril de 2013.

Aceito para a publicação em 26 de junho de 2013.

Parte da Dissertação de Mestrado do primeiro autor.

${ }^{2}$ Programa de Pós-Graduação em Sanidade, Segurança Alimentar e Ambiental no Agronegócio, Instituto Biológico, Av. Conselheiro Rodrigues Alves 1252, Vila Mariana, São Paulo, SP 04014-002, Brasil. *Autor para correspondência: luisgustavet@yahoo.com.br

${ }^{3}$ Embriza Biotecnologia Ltda, Rua Alberto Simões Pires 100, Vila Morumbi, Campo Grande, MS 79052-060, Brasil. E-mail: zanenga@embriza. com.br
}

RESUMO.- 0 uso do conhecimento de biotécnicas para a melhoria da produção de bens e serviços tem avançado significativamente. 0 controle de doenças infecciosas continua a representar o maior obstáculo para a saúde dos animais. A perda embrionária pode ser responsável pelo aumento de prejuízos econômicos isolados para os produtores de bovinos. A infecção por Neospora caninum tem emergido como uma importante doença reprodutiva e, em vários países, tem sido diagnosticada como principal causa de aborto. 0 
objetivo desse trabalho foi comparar o desempenho reprodutivo em receptoras bovinas não contaminadas e naturalmente infectadas por Neospora caninum após a transferência de embriões (TE). A escolha dos animais $(n=395)$ para o experimento foi realizada através de avaliação ginecológica, via palpação transretal. As vacinações seguiram protocolo sanitário recomendado para a TE. 0 manejo nutricional foi baseado no National Research Council (NRC, 1999). A TE de embriões descongelados foi feita após a sincronização do ciclo estral das receptoras. Para a avaliação do desempenho reprodutivo se utilizou de ultrassonografia transretal. A identificação das receptoras positivas para Neospora caninum realizou-se através do teste de ELISA e, para as amostras reagentes, confirmação através de imunofluorescência indireta (IFI). Os dados foram analisados utilizando Past $^{\circledR}$ e foram considerados estatisticamente significantes $(\mathrm{P}<0,01)$. Os resultados mostram uma taxa de prenhes ( $\mathrm{n}=191)$ de 48,35\%, seguida de uma taxa de abortos aos 60 dias de gestação (n=14) de 3,5\% e abortos ao sexto mês de gestação $(n=12)$ de 3,04\%. Esses resultados tornam-se relevantes quando se leva em consideração que, receptoras infectadas pelo protozoário não apresentaram uma taxa elevada de perdas gestacionais. A insignificância estatística dos dados obtidos nesse estudo confere a segurança em sugerir que não há necessidade em implantar o diagnóstico ou controle prévios para Neosporose em rebanhos aptos a TE.

TERMOS DE INDEXAÇÃO: Neosporose, Neospora caninum, aborto, concepção, receptoras de embriões, bovinos.

\section{INTRODUÇÃO}

O controle das doenças infecciosas continua a representar o maior obstáculo para a saúde animal. Prevenir doenças é mais econômico e eficaz do que tratar surtos que venham ocorrer. Portanto, a adoção de um programa preventivo de controle de doenças e infecções é essencial para a manutenção da saúde dos animais (Miyazaki \& Bôas 2007) e maximização da produção de uma propriedade.

A perda embrionária pode ser responsável por grandes quedas econômicas isoladas para os produtores de bovinos. No Brasil, com mais de 70 milhões de vacas de corte anualmente em reprodução, os prejuízos causados pela mortalidade embrionária podem chegar a 4 bilhões de reais (Santos \& Vasconcelos 2010).

Neospora caninum é facilmente transmitido em bovinos. Tanto a transmissão horizontal quanto a transmissão vertical são importantes rotas no desenvolvimento da infecção, vitais para a manutenção do parasita e circulação no hospedeiro (Dubey et al. 2006).

A transmissão horizontal ocorre quando um bovino ingere alimentos contaminados com os oocistos, na forma não esporulada, presentes nas fezes dos caninos (Dubey et al. 2004).

Já na transmissão vertical grande parte dos bezerros de vacas portadoras do protozoário, nascem infectados, porém clinicamente saudáveis, permitindo a manutenção do agente no rebanho por várias gerações (Dubey \& Lindsay 1996, Marley et al. 2006).

O sinal clínico mais importante da neosporose é o abor- to, ocorrendo entre o $5^{\circ}$ e $6^{\circ}$ mês de gestação, esporadicamente, endemicamente ou epidemicamente. As taxas de transmissão congênita podem chegar a ordem de 50 a 95\%, sendo uma importante via de manutenção do parasita no meio (Wouda et al. 1997, Scharles et al. 1998).

No Brasil, as taxas de sororeatividade são extremamente variáveis em rebanhos bovinos de leite e corte, na dependência do manejo, condições epidemiológicas, sensibilidade e especificidade dos testes diagnósticos empregados, com valores que variam de $8,4 \%$ a $44,8 \%$ (Pituco et al. 2001, Andreotti et al. 2002).

O objetivo desse trabalho foi comparar o desempenho reprodutivo em receptoras bovinas não contaminadas e naturalmente infectadas por Neospora caninum após a transferência de embriões.

\section{MATERIAL E MÉTODOS}

As receptoras ( $\mathrm{n}=392)$ são provenientes de fazendas dos estados de Minas Gerais (1980'57.46" S, 1955'80.92" 0) e São Paulo (2336'03.94" S, 4752'16.30" 0), que possuíam manejo envolvendo cães pastores. A avaliação ginecológica foi realizada através de palpação transretal, onde se avaliou o grau de tortuosidade da cérvix, espessura, simetria e contratilidade dos cornos uterinos além do tamanho, presença e/ou ausência de estruturas, como folículos e corpo lúteo, nos ovários.

Foram realizados exames para a detecção de brucelose e tuberculose, segundo as normas do Programa Nacional de Controle e Erradicação da Brucelose e Tuberculose e, somente os animais com resultados negativos para as enfermidades, foram utilizados. Todas as receptoras foram submetidas a primo-vacinação, na dose de $5 \mathrm{ml}$, contra Rinotraqueíte Infecciosa Bovina (IBR), Diarréia Viral Bovina (BVD), Parainfluenza tipo 3, Vírus Respiratório Sincicial Bovino (BRSV) e Leptospirose (CattleMaster ${ }^{\circledR} 4+$ L5) com reforço após 21 dias, por via intramuscular profunda, contra clostridioses (Convexin ${ }^{\circledR}$ 9), na dose de $3 \mathrm{ml}$, por via subcutânea e adicionou-se ao tratamento, Estreptomicina (Estreptomax ${ }^{\circledR}$ ), na dose de $50 \mathrm{mg} / \mathrm{kg}$ de peso vivo, por via intramuscular profunda, em dose única, usando seringas hipodérmicas descartáveis luer-lock $\left(\mathrm{BD}^{\circledR}\right)$, de uso único, com agulhas hipodérmicas descartáveis $21 \mathrm{G}\left(\mathrm{BD}^{\circledR}\right)$. As receptoras inovuladas pela primeira vez e que não tiveram a prenhez confirmada, receberam uma dose de vacina contra leptospirose (Leptoferm ${ }^{\circledR}$ Pfizer), na dose de $5 \mathrm{ml}$, por via intramuscular profunda, em dose única.

No período destinado a campanha nacional, realizou-se as vacinações, contra a Febre Aftosa (Oleovac ${ }^{\circledR}$ Aftosa) e raiva dos bovinos (Rai-Vac $\mathrm{K}^{\circledR}$ ), por via subcutânea, em dose única de $5 \mathrm{ml}$, respectivamente, utilizando seringa multidose $\left(\right.$ Hoppner $\left.^{\circledR}\right)$ e agulha $18 \times 20 \mathrm{~mm}\left(\mathrm{BD}^{\circledR}\right)$.

Para descartar qualquer possibilidade de prenhez em fase inicial de gestação e que, à palpação retal fosse imperceptível (gestação com idade inferior a 30 dias), administrou-se $150 \mu \mathrm{g}$ de D-Cloprostenol (Prolise ${ }^{\circledR}$ ), por via intramuscular profunda, utilizando seringa hipodérmica descartável luer-lock $\left(\mathrm{BD}^{\circledR}\right)$, de uso único, com agulhas hipodérmicas descartáveis $21 \mathrm{G}$.

Para a desverminação, utilizou-se $7,5 \mathrm{mg} / \mathrm{kg}$ de albendazol $\left(\right.$ Agebendazol $^{\circledR}$ ) e $200 \mathrm{mcg}$ de ivermectina 1\% (Ivermectina Ouro Fino $^{\circledR}$ L.A.), por via subcutânea, com seringa multidose (Hoppner ${ }^{\circledR}$ ) e agulha $18 \times 20 \mathrm{~mm}\left(\mathrm{BD}^{\circledR}\right)$.

As receptoras receberam, diariamente, em sistema intensivo, uma dieta balanceada (National Research Council - NRC 2009), contendo $70 \%$ de silagem de milho acrescida com $30 \%$ de pré-secado de azevém, como alimentação volumosa e $2 \mathrm{~kg}$ de qui- 
rera de milho como concentrado, até que atingissem 60 dias de gestação.

Após o $60^{\circ}$ dia de gestação, as receptoras foram transferidas para uma propriedade secundária, localizada na cidade de Capitólio/MG (20³9'05.83"S, 4605'56.58”0), caracterizada por sistema extensivo de criação, contendo piquetes de Brachiaria brizantha cv. Marandú.

Nos piquetes, das duas propriedades, o fornecimento de sal mineral, contendo $88 \mathrm{~g}$ de fósforo (Fosbovi $20^{\circledR}$ ), ocorreu ad libitum.

A classificação do escore de condição corporal ocorreu em uma escala de 1 (animais magros) a 5 (animais obesos), segundo Otto et al. (1999), excluindo-se a interferência desse para os resultados obtidos no grupo experimental, sendo utilizados animais que mantiveram-se entre os escores entre 3 e 4, considerados como animais aptos a reprodução.

Para a sincronização do ciclo estral, no dia zero foi introduzido o implante intravaginal de liberação lenta de progesterona (Primer ${ }^{\circledR}$ ), com a utilização de um aplicador próprio (Primer ${ }^{\circledR}$ ) e por via intramuscular profunda com seringa hipodérmica estéril luer-lock $\left(\mathrm{BD}^{\circledR}\right)$ e agulha hipodérmica estéril $21 \mathrm{G}\left(\mathrm{BD}^{\circledR}\right)$, foi aplicado $2 \mathrm{mg}$ de benzoato de estradiol (Ric-Be ${ }^{\circledR}$ ). No dia 7, aplicou-se 300 UI de Gonodotrofina Coriônica Equina (eCG) (Folligon ${ }^{\circledR}$ ) e $150 \mu \mathrm{g}$ de D-Cloprostenol (Prolise ${ }^{\circledR}$ ), por via intramuscular profunda, com seringa hipodérmica estéril luer-lock $\left(\mathrm{BD}^{\circledR}\right)$ e agulha hipodérmica estéril $21 \mathrm{G}\left(\mathrm{BD}^{\circledR}\right)$. No dia 9, retirou-se o implante intravaginal e, posteriormente, aplicou-se $1 \mathrm{mg}$ de benzoato de estradiol (Ric-Be $\left.{ }^{\circledR}\right)$, com seringa hipodérmica estéril luer-lock $\left(\mathrm{BD}^{\circledR}\right.$ ) e agulha hipodérmica estéril $21 \mathrm{G}\left(\mathrm{BD}^{\circledR}\right)$. Esse procedimento foi realizado individualmente em todas as receptoras submetidas ao processo de transferência de embriões em tempo fixo (TETF).

Após 18 dias do início da sincronização, os embriões foram descongelados, avaliados segundo as normas da Sociedade Internacional de Tecnologia de Embriões (Iets 2010) e transferidos, em tempo fixo, nas receptoras, por via transcervical, após anestesia epidural baixa sacrococcígea com $5 \mathrm{ml}$ de lidocaína $2 \%$ (Lidovet $\left.^{\circledR}\right)$, utilizando seringa hipodérmica descartável estéril luer-lock $\left(\mathrm{BD}^{\circledR}\right)$ e agulha hipodérmica descartável estéril 21G.

Apenas um embrião foi depositado, o mais próximo possível do ovário ipsilateral ao corpo lúteo de cada receptora.

Todos os embriões utilizados foram cedidos pela Embriza Biotecnologia Ltda.

As palhetas contendo os embriões foram descongeladas após 10 segundos em atmosfera ambiente e 20 segundos submersas em água aquecida entre 30 e $36^{\circ} \mathrm{C}$.

Após secagem das palhetas em papel toalha, as peças de obliteração foram removidas e os embriões retirados de seu interior. Os mesmos foram levados a uma placa de quatro poços (Nunc ${ }^{\circledR}$ ) com três diferentes diluições de glicerol (Nutricell ${ }^{\circledR}$ ), onde permaneceram por 5 minutos em cada banho e, logo após, foram lavados em solução de manutenção (TQC-Holding, Nutricell ${ }^{\circledR}$ ). A observação foi realizada em um esteromicroscópio binocular, com aumento de 80 vezes, (Nikon ${ }^{\circledR}$ SMZ-645).

Posteriormente, os embriões foram envasados, com o auxílio de uma seringa hipodérmica descartável estéril de $1 \mathrm{ml}\left(\mathrm{BD}^{\circledR}\right)$, em paietes de $0,25 \mathrm{ml}$ (Nutricell ${ }^{\circledR}$ ) e colocados em bainha estéril $\left(\mathrm{WTA}^{\circledR}\right)$ no aplicador universal para transferência dos embriões $\left(\mathrm{WTA}^{\circledR}\right)$.

Após 30 dias da TETF, realizou-se o diagnóstico da gestação por ultrassonografia, com sonda transretal linear com $5 \mathrm{MHZ}$ de frequência (Mindray ${ }^{\circledR} 2200$ VET). As receptoras que não emprenharam foram submetidas a um novo protocolo de sincronização e TETF. Quando, pela segunda vez, não tiveram a gestação confirmada, foram abatidas em frigorífico credenciado pelo Serviço de Inspeção Federal.
As receptoras prenhes aguardaram até 60 dias pós-transferência para confirmação da gestação e sexagem fetal, por meio de ultrassonografia, com sonda transretal linear com frequência de 5 MHZ, (Mindray ${ }^{\circledR} 2200$ VET). Então, foram transportadas até uma propriedade secundária, situada na cidade de Capitólio/MG, onde permaneceram até 180 dias de gestação. Nesse período gestacional, novamente foram avaliadas ginecologicamente, por palpação transretal, para diagnóstico de possíveis perdas fetais.

Coletados todos os dados, as receptoras foram separadas em grupos positivos e negativos para o agente. Desses, foram comparados os dados relativos a taxas de prenhez e taxas de abortos.

A identificação das receptoras positivas para o $N$. caninum ocorreu por meio de colheita de sangue da veia caudal mediana, utilizando tubo com vácuo sem anticoagulante (Vacutainer ${ }^{\circledR}$ ) e agulha hipodérmica descartável múltipla para coleta 21G (Vacutainer $\left.^{\circledR}\right)$. Após a centrifugação e o congelamento, em criotubos de $2 \mathrm{ml}$ (Corning ${ }^{\circledR}$ ), identificados individualmente com o número de cada receptora, as amostras foram submetidas ao teste de ELISA indireto (Idexx ${ }^{\mathrm{TM}}$ Laboratories) e, para as receptoras reagentes, a confirmação ocorreu através da reação de imunofluorescência indireta (IFI) no Laboratório de Viroses de Bovídeos, do Instituto Biológico em São Paulo, sendo considerados reagentes aquele que apresentaram títulos iguais ou superiores a 1:200.

Como modelo estatístico utilizou-se o programa Past ${ }^{\circledR}$ para a análise multivariada dos dados (Cluster Analysis) e a Análise dos Componentes Principais (PCA), com $\mathrm{P}<0,01$.

\section{RESULTADOS}

A proporção de animais gestantes e não gestantes positivos e negativos (Quadro 1), para o Neospora caninum, não diferiram estatisticamente $(\mathrm{P}>0,01)$.

Quando as taxas de abortos foram comparadas entre os grupos sororreagentes (Quadro 2), não se observa diferença estatística $(\mathrm{P}>0,01)$ entre os animais que são positivos e negativos para Neospora caninum, corroborando a insig-

Quadro 1. Diagnóstico de gestação após a detecção de soropositividade por Neospora caninum através das técnicas de ELISA e RIFI nas receptoras de embriões com diferentes idades gestacionais

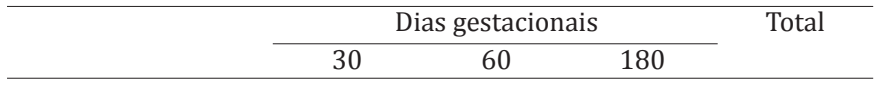

Neospora po- Prenhe $13(4,36 \%) 12(4,02 \%) 11(3,69 \%) 36(37,50 \%)$ sitivo (n=96) Vazia $19(6,37 \%) 19(6,37 \%) 20(6,47 \%) 58(60,41 \%)$ Aborto $\quad 0(0 \%) \quad 1(0,33 \%) \quad 1(0,33 \%) \quad 2(2,08 \%)$

Neospora ne- Prenhe $15(5,03 \%) 11(3,69 \%) 8(2,68 \%) 34(50,74 \%)$ gativo (n=67) Vazia $7(2,34 \%) \quad 8(2,68 \%) \quad 11(3,69 \%) 26(38,80 \%)$ Aborto $1(0,33 \%) \quad 3(1 \%) \quad 3(1 \%) \quad 7(10,44 \%)$

${ }^{*} \mathrm{P}<0,01$ : Cluster Analysis e Análise dos Pontos Principais (Past ${ }^{\circledR}$ ).

Quadro 2. Diagnóstico de gestação após a detecção de anticorpos anti-Neospora caninum, pela técnica de ELISA, nas receptoras de embriões com diferentes idades gestacionais

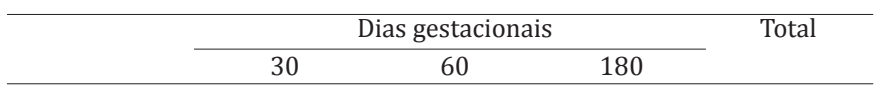

Sorone- Prenhe 137 (45,97\%) 126 (42,28\%) 118 (39,59\%) 118 (48,55\%) gativos

Sorone- Vazia $106(35,57 \%) 106(35,57 \%) 106(35,57 \%) 106(43,62 \%)$ gativos

Sorone- Aborto $\quad 0(0 \%) \quad 11(3,69 \%) \quad 8(2,68 \%) \quad 19(7,8 \%)$

gativos

* P<0,01: Cluster Analysis e Análise dos Pontos Principais (Past®). 
nificância do agente em relação às perdas gestacionais nas diferentes idades gestacionais avaliadas. Quanto às taxas de prenhez, também, não houve significância dos dados.

Em todas as receptoras soronegativas no exame de ELISA indireto (Quadro 2, Fig.1), nos diferentes grupos (prenhes, vazias e abortos) entre as diferentes idades gestacionais, não se observou qualquer diferença estatística $(\mathrm{P}>0,01)$.

Quando comparadas as taxas de abortos aos 180 dias entre os grupos que são sororreagentes $(5,5 \%)$ e os soronegativos $(7,8 \%)$, não se observa qualquer diferença estatística $(\mathrm{P}>0,01)$.

Entre os animais sororreagentes, 2,4\% (n=4) abortaram aos 180 dias de gestação, caracterizando a insignificância estatística $(\mathrm{P}>0,01)$ para animais positivos em relação às perdas gestacionais.

De acordo com a frequência de ocorrência de Neospora caninum (Quadro 3.) dos animais analisados, observou-se que $11,04 \%$ das receptoras analisadas obtiveram resultados positivos para a neosporose e, dessas, uma receptora $(0,33 \%)$ abortou entre 30 e 60 dias de gestação e outra abortou ao sexto mês gestacional $(\mathrm{P}>0,01)$.

Com relação à ocorrência de receptoras soronegativas para neosporose, 2,68\% apresentaram abortos no período compreendido entre 60 e 180 dias de gestação, sugerindo

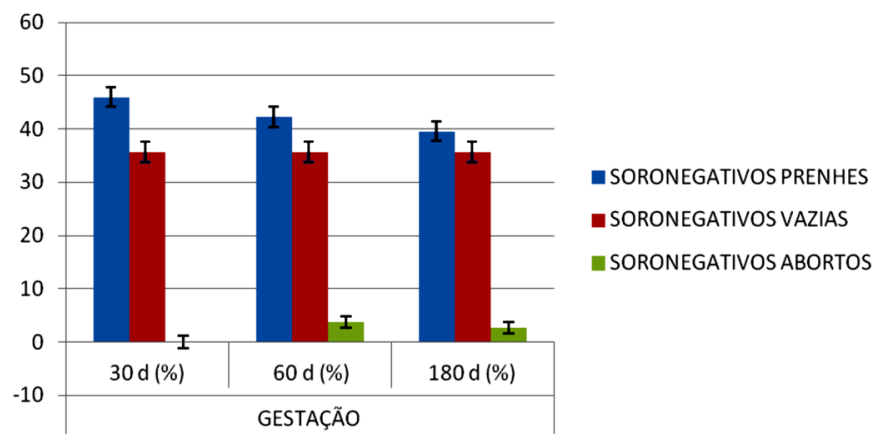

Fig.1. Diagnóstico de gestação após a detecção de anticorpos anti-Neospora caninum, pela técnica de ELISA, nas receptoras de embriões com diferentes idades gestacionais.

Quadro 3. Frequência da ocorrência de Neospora caninum nas diferentes idades gestacionais de receptoras sororeagentes positivas e negativas para ELISA e confirmados pela RIFI e soronegativas para o agente

\begin{tabular}{|c|c|c|c|c|}
\hline Variável & Dias de & Frequência de so & eagentes ELISA* & Frequência de \\
\hline Prenhez & gestação & Positivos & Negativos & soronegativos \\
\hline & & RIFI & RIFI & ELISA* \\
\hline Prenhe & 30 & $13(4,36 \%)$ & $15(5,03 \%)$ & $137(45,97 \%)$ \\
\hline & 60 & $12(4,02 \%)$ & $11(3,69 \%)$ & $126(45,28 \%)$ \\
\hline & 180 & $11(3,69 \%)$ & $8(2,68 \%)$ & $118(39,59 \%)$ \\
\hline Vazia & 30 & $19(6,37 \%)$ & $7(2,34 \%)$ & $106(35,57 \%)$ \\
\hline & 60 & $19(6,37 \%)$ & $8(2,68 \%)$ & $106(35,57 \%)$ \\
\hline & 180 & $20(6,47 \%)$ & $11(3,69 \%)$ & $106(35,57 \%)$ \\
\hline Aborto & 30 & $0(0 \%)$ & $1(0,33 \%)$ & $0(0 \%)$ \\
\hline & 60 & $1(0,33 \%)$ & $3(1 \%)$ & $0(0 \%)$ \\
\hline & 180 & $1(0,33 \%)$ & $3(1 \%)$ & $8(2,68 \%)$ \\
\hline TOTAL & & $96(11,04 \%)$ & $67(7,71 \%)$ & $707(81,36 \%)$ \\
\hline & & $163(18,76 \%)$ & & \\
\hline & & 869 (100\%) & & \\
\hline
\end{tabular}

* P<0,01: Cluster Analysis e Análise dos Pontos Principais (Past®). que o estresse caracterizado pelo transporte entre as propriedades, com distância aproximada de $860 \mathrm{~km}$, tenha provocado tais perdas.

Durante todo o período desde a transferência dos embriões até o sexto mês de gestação as taxas de abortos dos animais soronegativos em relação aos animais soropositivos, foi de 2,10\% (P>0,01).

\section{DISCUSSÃO}

No Brasil, as taxas de sororreatividade são extremamente variáveis em rebanhos bovinos de leite e corte, devido ao tipo de manejo adotado, condições epidemiológicas, sensibilidade e especificidade dos testes diagnósticos empregados, com valores que variam de $8,4 \%$ a 44,8\% (Pituco et al. 2001, Andreotti et al. 2002).

Em estudos realizados por Yániz et al. (2008) as taxas de abortos, em vacas de alta produção soropositivas, relacionadas com soronegativas para Neospora caninum, foram de $23,6 \%$ e $2,3 \%(\mathrm{P}<0,05)$, respectivamente.

A presença de anticorpos anti-Neospora caninum e a ocorrência de abortamentos em 188 vacas pluríparas de rebanhos leiteiros da região Noroeste do Estado de São Paulo, revelou que o agente está presente em 37,6\% dos animais e que existe elevada associação entre a presença de anticorpos anti-N. caninum e abortamento (Stobbe 1999). A frequência de ocorrência de animais sororreagentes com idade superior de seis meses foi de 5,6\% segundo Del Fava et al. (2007).

Andreotti et al. (2010), utilizando novilhas no Pantanal Sul-Matogrossense, observaram que animais que não emprenharam, possuíam soroprevalência 22,13\% maior do que as que conceberam, indicando que há uma correlação significativa entre a não concepção e a presença de anticorpos anti-Neospora caninum e verificaram alto impacto na reprodução, sendo o índice de gestação de 44,11\% para vacas soropositivas e 59,12\% para vacas soronegativas $(\mathrm{P}<0,05)$.

Segundo Moore et al. (2003) comparando o efeito das perdas reprodutivas em rebanhos de corte na Argentina por N. caninum $x$ Tritrichomonas foetus, constataram que todos os abortos registrados foram causados por N. caninum.

Porém López-Gatius et al. (2005) avaliaram três rebanhos leiteiros com média de $12 \%$ de fêmeas soropositivas e constataram que a taxa de prenhez foi de $34 \%$ nas vacas soronegativas e $32 \%$ nas soropositivas. Logo, esses autores, afirmam que a infecção por Neospora caninum não interferiu na fertilidade.

Em estudo realizado por Del Fava et al. (2007) a sororreatividade para $N$. caninum não apresentou associação com os índices de prenhez de reagentes $(83,3 \%)$ e não reagentes $(73,9 \%)(p>0,05)$ e, tão pouco, nas taxas de parição de reagentes $(85,0 \%)$ e não reagentes $(83,3 \%)(p>0,05)$. A condição corporal e o peso de vacas gestantes ou não gestantes e paridas ou não paridas $(p>0,05)$ não sofreu interferência de $N$. caninum. Concluiu-se que no rebanho estudado, nas condições climáticas e de manejo adotadas, que os índices de prenhez e parição, bem como as características de desempenho não apresentaram associação com a sororeatividade para $N$. caninum. 
De acordo com Paz et al. (2007) a frequência de soropositividade de $N$. caninum foi de $29,5 \%$ demonstrando alta incidência desse agente no rebanho estudado. Quando o desempenho reprodutivo foi levado em consideração, a taxa de prenhez observada no grupo de animais soropositivos foi de $72,7 \%$ e do grupo soronegativo foi de $81,8 \%$, não apresentando diferença significativa dos resultados $(\mathrm{P}>0,05)$.

Anticorpos de $N$. caninum e o parasita em, aproximadamente, um terço de todas as vacas de leite testadas, foram identificados entre $32 \%$ a $57 \%$ de todos os fetos bovinos abortados (Mainar-Jaime et al. 1999, Pereira-Bueno et al. 2003).

\section{CONCLUSÕES}

Os resultados obtidos demonstram que a frequência de ocorrência de animais soropositivos em relação aos soronegativos para Neospora caninum, que gestaram ou não, acabaram não diferindo estatisticamente.

E quando consideradas somente as receptoras soronegativas para o protozoário, que gestaram e que não gestaram, não foi observada qualquer significância nos resultados.

Portanto, nesse estudo, com dados estatísticos insignificantes, é seguro sugerir que não há necessidade de implantar um diagnóstico ou controle prévio para neosporose em rebanhos aptos a transferência de embriões. Essa insignificância estatística, também se aplica na proporção de animais gestantes e não gestantes positivos e negativos para $N$. caninum em relação aos animais negativos para o protozoário.

\section{REFERÊNCIAS}

Andreotti R., Pinckney R.D., Pires P.P. \& Silva E. 2002. Serology of antiNeospora caninum in beef cattle and in dogs in the state of Mato Grosso do Sul, Central Brazil. Anais XII Congresso Brasileiro de Parasitologia Veterinária, Rio de Janeiro.

Dubey J.P. \& Lindsay D.S. 1996. A review of Neospora caninum and neosporosis. Vet. Parasitol. 67(1):1-59.

Dubey J.P., Sreekumar C., Knickman E., Miska K.B., Vianna M.C.B., Kwok O.C.H., Hill D.E., Jenkins M.C. \& Lindsay D.S. \& Greene C.E. 2004. Biologic, morphologic, and molecular characterization of Neospora caninum isolates from littermate dogs. Int. J. Parasitol. 34:1157-1167.
Dubey J.P., Buxton D. \& Wouda W. 2006. Pathogenesis of bovine neosporosis. J. Comp. Pathol. 134(4):267-289.

Mainar-Jaime R.C., Thurmond M., Berzal-Herranz B. \& Hietala S. 1999. Seroprevalence of Neospora caninum and abortion in dairy cows in northern Spain. Vet. Rec. 145:72-75.

Maley S.W., Buxton D., Rae A.G., Wright S.E., Schock A., Bertley P.M., Esteban-Redondo I., Swales C., Hamilton C.M., Sales J. \& Innes E.A. 2006. The pathogenesis of neosporosis in pregnant cattle: inoculation at Midgestation. J. Comp. Pathol. 129(2/3):186-195.

Miyazaki N.H.T. \& Bôas M.H.S.V. 2007. Medidas sanitárias empregadas na criação de animais, p.165-185. In: Cardoso T.A.O. \& Navarro M.B.M.A. (Eds), A Ciência entre Bichos e Grilos: reflexões e ações da biossegurança com animais. FAPERJ, Rio de Janeiro.

Otto K.A., Ferguson J.D., Fox D.G. \& Sniffen C.J. 1991. Relationship between body condition score and composition of 9-10-11 rib tissue in Holstein dairy cows. J. Dairy Sci., 74:852-859.

Paz G.F., Leite R.C. \& Rocha M.A. 2007. Associação entre sorologia para Neospora caninum e taxa de prenhez em vacas receptoras de embriões. Arq. Bras. Med. Vet. Zootec. 59(5):1323-1325.

Pereira-Bueno J., Quintanilla-Gozalo A., Perez-Perez V., Espi-Felgueroso A., Alvarez-Garcia G., Collantes-Fernandez E. \& Ortega-Mora L.M. 2003. Evaluation by different diagnostic techniques of bovine abortion associated with Neospora caninum in Spain. Vet. Parasitol. 111:143-152.

Pituco E.M., Stefano E., Okuda L.H., Trotter C.M., Perucini L.M. \& Duarte F.C. 2001. Sorodiagnóstico de neosporose bovina no Brasil. Anais 14aㅡ Reunião Anual do Instituto Biológico, São Paulo.

Santos R.M. \& Vasconcelos J.L.M. 2010. Estratégias para reduzir perdas embrionárias. 1. <www.beefpoint.com.br> Acessado em 20 fev. 2010.

Schares G., Peters M., Wurm R., Bärwald A. \& Conraths F.J. 1998. The efficiency of vertical transmission of Neospora caninum in dairy cattle analysed by serological techniques. Vet. Parasitol. 80(2):87-98.

Stobbe N.S. 1999. Estudo interativo entre a presença de anticorpos anti-Neospora caninum e a ocorrência de abortamento em bovinos no Noroeste do Estado de São Paulo, Brasil. Tese de Doutorado, Faculdade de Medicina Veterinária e Zootecnia, Universidade de São Paulo, São Paulo, SP.

Wouda W., Moen A.R., Visser I.J. \& Van Knapen F. 1997. Bovine fetal neosporosis: a comparison of epizootic and sporadic abortion cases and different age classes with regard to lesion severity and immunohistochemical identification of organisms in brain, heart, and liver. J. Vet. Diagn. Invest. 9:180-185.

Yániz J.L., López-Gatitus F., García-Ispierto I., Bech-Sàbat G., Serrano B., Nogareda C., Sanchez-Nadal J.A., Almeria S. \& Santolaria P. 2008 Some factors affecting the abortion rate in dary herds with high incidence of Neospora-associated abortions are different in cows and heifers. Reprod. Dom. Anim. 45(4):699-705. 